\title{
No new ways of treating AIDS
}

\section{The discovery of what may be the agent responsible for the simian analogue of AIDS does not, for the time being, help with understanding the human disease or simplify its management.}

\section{San Francisco}

ONE way and another, this splendid city has the dubious reputation of being the AIDS capital of the world. Although new cases now arise more frequently elsewhere, in New York for example, this is where the unusual occurrence of Kaposi's sarcoma and lung infection by the organism Pneumocystis carinii were first recognized to be symptoms of an underlying profound defect of the immune system now known as acquired immune deficiency disease, or AIDS. What follows is an account of a brief conversation with some of the staff at the clinic set up at the San Francisco General Hospital for the treatment of patients with the disease.

The first thing to say is how little there is to say. The second, that in the circumstances it is remarkable that the staff of the clinic (mostly physicians in their thirties) should so quickly have cultivated such an admirable blend of detachment and understanding in the face of the frustration which is their daily lot.

Even the news from the Davis campus of the University of California that $\mathrm{Dr}$ Preston L. Marx's group there has identified a B-type retrovirus as the probable causative agent of the simian (monkey) analogue of human AIDS seems to offer no relief from the frustration. That report, said to have appeared in the current issue of Science, does not, after all, suggest that the causative agents of simian and human AIDS are identical or even prove that the agent of human AIDS is a retrovirus. People here are keen to point out that a DNA virus with properties akin to those of hepatitis-B virus would equally explain the tendency of the human AIDS virus to go antigenically to ground.

For what it is worth, the agent of simian AIDS seems to have been about for longer than its human analogue. Although the retrovirus identified at Davis is derived from a rhesus monkey that died with immune deficiency more than a year ago (and although it has since been cultured in monkey kidney cells and also transmitted to other monkeys), Dr Marx thinks it likely that evidence for its occurrence can be found in the records of the rhesus monkey colony going back at least to 1975 , perhaps even earlier. The proof that the virus is a retrovirus hangs on its shape (seen in electron micrographs) and on the presence of reverse transcriptase (for converting RNA into the appropriate DNA) in tissue cells which harbour it. The difficulty, for physicians, is that even if the agent responsible for human AIDS may be similar in kind, there is no obvious way in which this can be demonstrated quickly and no obvious way, thereafter, to the development of prophylactic techniques.

So how gloomy should one be about human AIDS? The physicians note that the treatment they are at present able to provide for their patients in the clinic amounts to nothing but the treatment of the adventitious symptoms of the underlying condition. And even there, the prospects for intervention are poor. Drugs that could be counted on to succeed in the treatment of, say, Pneumocystis carinii infection in healthy patients turn out to be toxic in patients with AIDS. So it may be necessary to treat a patient suffering from such an infection with two drugs consecutively in the knowledge that this will exhaust the repertoire of what is available, meaning that a second bout of infection may be intractable.

This is why immediate hopes centre on the trials about to be mounted in San Francisco of the efficacy of genetically engineered $\gamma$-interferon and of interleukin-2. Both studies should be under way within six weeks. There is also a study of the usefulness of $\alpha$-interferon among people suffering from blood-cell disorders that may be precursors of outright AIDS but which may also be less severe consequences of infection by the same causative agent.

What, in the circumstances, can patients suffering from AIDS be told? The simple answer seems to be the truth. The physicians point out that the population of male homosexuals most at risk is also, in San Francisco, an intelligent population of people able and willing to assume responsibility for their own fate. Patients therefore get to know that if they are properly diagnosed as suffering from AIDS, the prognosis is gloomy. Many take the view that the best they can do is to arrange that the quality of their life shall be as far as possible preserved. Hospitalization is wherever possible avoided.

So how serious is the outbreak of human AIDS? And is it fair to describe it as an epidemic? While the number of cases so far recognized is still only just over 400 , people are quick to point out that the incidence of the disease is high among the population at risk, perhaps several hundred a year per 100,000 single men. Moreover, there seems no sign of a decline in the rate at which cases accumulate in the city, which appears to have increased month by month except for a brief hiatus in mid-1983. Sooner or later, of course, the rate at which new cases occur should level off, but when that will be can be told only when more known of the natural history of the disease and of how the infection is acquired.

Epidemic is, then, the right word; that is what the physicians say. Indeed, part of their anxiety is that the population at risk may be lulled into a false sense of security by optimistic reports that some new development may spell the understanding and imminent treatment of the disease.

Indeed, they have a still more chilling vision of where the present epidemic may lead. The agent of AIDS, whatever it is, seems to be in its virulent form a genuinely novel infection of human beings. Since the condition was first recognized for what it is, there has naturally been a diligent search of past medical records for evidence of the characteristic adventitious symptoms, among which Kaposi's sarcoma should be the most distinctive. So far, the search has been unsuccessful. So what, in 1979, when the first deaths occurred from what was two years later recognized as AIDS, can have happened to the causative agent? The analogy with the agent of syphilis, a skin disease before it became a venereal disease, shows that infectious agents can radically change their habit and the nature of the disease they cause. May, then, the agent of AIDS change again, becoming in the process a more generalized infection of people?

The opinion that the AIDs agent may be an infectious agent in the course of adaptation to a new ecological relationship with human beings is supported by evidence as well as by imagination. The difference between the symptoms manifest among male homosexuals and among the others groups among whom the disease occurs, emigrants from Zaire or Haiti, for example, suggests some such variability.

Others working in the field give a different but no less chilling account of what is happening. Noting the prevalence of lymphadenopathy among the population at risk which appears not always to become frank AIDS, they imply that the infectious agent may already be widespread in the human population, in which case the objective of research should be not so much to identify the virus as to tell what trauma makes infection become so damaging.

John Maddox 